\title{
Los sondeos de Clinton \\ y las paradojas de la democracia
}

\author{
JUAN JESÚs GONZÁLEZ \\ Cal Poly, California \\ Marzo de 2001
}

\section{INTRODUCCIÓN}

Las paradojas de la democracia son bien conocidas: los gobiernos están para gobernar y los líderes para dirigir, pero se espera de ellos que gobiernen de acuerdo con la opinión de los electores, es decir que dirijan el curso de los acontecimientos sin apartarse de los deseos populares. El problema es que una de dos: o son realmente líderes, en cuyo caso les sobrarán razones para apartarse de los deseos populares (no solo por la naturaleza de algunos temas, pongamos por caso la politica fiscal, sino por el inevitable gap de información que se produce entre electores y elegidos), o se limitan a seguir la corriente de la opinión pública, en cuyo caso se comportarán como seguidores oportunistas más que líderes.

Los líderes tienen poco margen de maniobra cuando la gente considera que han sido designados para cumplir un mandato. Tendrán mucho más, en cambio, si la gente considera que se les ha dado un voto de confianza. Que sea de una u otra manera depende fundamentalmente de dos cosas: de la coyuntura política y del perfil de los electores. Pero salvo coyunturas excepcionales y salvo electores muy ideologizados, los electores en general no eligen tanto a los políticos para que hagan cosas concretas, como para que las hagan bien -las que haya que hacer en cada momento. De ahí que la decisión de voto no descanse tanto en una proyección de expectativas a partir de los programas que presentan los partidos

NotA: este artículo ha sido elaborado durante una estancia sabática en el Departamento de Ciencias Sociales de la Universidad Politécnica de California (Cal Poly), financiada por el Vicerrectorado de Investigación de la UNED. El autor agradece el apoyo tanto del Vicerrectorado como del citado Departamento. Y agracede también los comentarios de Miguel Caínzos, Miguel Requena y Nancy Konvalinka en relación con la traducción de algunos términos. 
como en una evaluación retrospectiva de la actuación de los partidos y, en particular, del partido gobernante. Y de ahí que el voto funcione mucho más como instrumento de control que como indicación de mandato.

¿Qué papel juegan los sondeos en este contexto? Caben varias posibilidades: si los políticos se sienten constreñidos por un mandato, hay poco margen para los sondeos. La política sería en tal caso una cuestión de principio: cumplir o no cumplir, esa es la cuestión. Pero si los políticos reciben un voto de confianza, los sondeos serían no solo recomendables, sino un imperativo del buen funcionamiento de la democracia. Pues no se trataría solo de que los políticos estuviesen al corriente de los deseos y las expectativas populares, sino de que las incorporen a su agenda en los términos más precisos.

Cabrían en este caso dos posibilidades: que esta incorporación se hiciese, por así decir, al pie de la letra o que diese lugar a un diálogo con la opinión pública en virtud del cual los políticos ajustasen sus agendas en sintonía con la opinión pública. En este punto, suelen dispararse las alarmas de los lectores temerosos de que un argumento así encierre una nueva justificación, quizá más sofisticada, de la capacidad de los partidos para manipular la opinión pública. ¿Pues no llegaríamos por esa vía al punto en que los sondeos y focus groups no sirven sino para proporcionar a los políticos el equivalente del rayo láser con que teledirigir sus bombas inteligentes? En efecto, no conviene en ningún caso descartar esta tercera posibilidad, pero creo que merece la pena explorar la posibilidad de diálogo entre los partidos y la opinión pública.

A tal fin, voy a comenzar argumentando la idea del voto como depósito de confianza, el cual obliga a los políticos a rendir cuentas de su gestión al término de su mandato. Aunque los programas electorales cumplen una función insustituible en la orientación de dicha gestión, los sondeos juegan un papel cada vez más importante en la implementación de estos programas, alimentando un proceso permanente de (re)elaboración de las agendas políticas de los partidos. Desde este punto de vista, los programas de los partidos pueden ser entendidos como declaraciones de principio cuya aplicación práctica queda a expensas de los ajustes resultantes del diálogo con la opinión pública, de manera que los programas han de pasar el doble filtro de las urnas y de los sondeos.

La razón de este doble filtro es fácil de entender: el voto no tiene ninguna capacidad deliberativa, es tan solo un depósito de confianza que no nos dice nada de la opinión de los electores sobre los aspectos concretos de la acción de gobierno. En justa coherencia, los electores delegan responsabilidad y evaluan la actuación de los partidos a posteriori. Esto tiene dos tipos de implicaciones: por un lado, hace que los partidos estén más pendientes del control posterior que del mandato previo -de ahí que estén sumamente pendientes del curso de la opinión pública. Por otro, hace que los votantes estén más atentos de lo que hacen los partidos que de lo que prometen -de ahí la superioridad explicativa de las teorías retrospectivas del voto.

A continuación, voy a plantear las posibles estrategias de los partidos ante la opinión pública. Justamente lo llamativo de algunos estudios recientes sobre el rendimiento de la democracia es el reflujo de lo que se consideraba una tendencia secular hacia la mayor transparencia y la sensibilidad democrática de los partidos. Esto es claro en el caso de EEUU, de acuerdo con Jacobs y Shapiro (2000), 
donde los años ochenta y noventa han registrado un declive significativo en la capacidad de la clase política para acomodarse a la opinión pública. Esto ha sido así pese a severas derrotas electorales tanto republicanas como demócratas, lo que hace suponer a estos autores que «el castigo electoral puede no ser suficiente para mejorar la influencia del público sobre el gobierno» (2000: xvii).

Por supuesto, no se trata de una tendencia lineal, sino de una tendencia condicionada por la distancia respecto de las elecciones, de manera que dentro de cada legislatura o mandato se puede observar una recuperación de la sensibilidad de los partidos a medida que se acerca el momento electoral.

\section{LA DEMOCRACIA COMO DEPÓSITO DE CONFIANZA}

El reciente estudio de Manin, Przeworsky y Stokes (1999) nos proporciona un buen punto de partida para nuestra discusión. Dichos autores parten de la distinción entre dos maneras de entender la representación democrática: como mandato o como control. La idea de representación como mandato opera sobre el doble supuesto de que la acción de los gobiernos sigue fielmente sus promesas electorales y de que estas se ajustan a los intereses de los electores. El problema es que las condiciones que definen lo que es mejor para los electores son cambiantes y aunque los votantes pueden disgustarse con gobiernos que abandonan sus promesas, es poco probable que los castiguen si observan que su bienestar mejora con ello (op cit: $35 \mathrm{ss}$ ). De manera que si bien es cierto que los partidos que ganan elecciones de manera contundente tienden a presentarse como portadores de un mandato imperativo, el propio curso de la acción de gobierno suele recomendar cautela sobre su aplicación práctica.

La idea de la democracia como forma de control opera, en cambio, sobre el simple supuesto de que los electores reeligirán a los gobernantes si su actuación consigue una evaluación favorable. En ocasiones, no hace falta que sea una valoración positiva, basta con que los electores estén convencidos de que la oposición no lo hubiera hecho mejor. De ahí las diversas posibilidades de que la citada evaluación incluya juicios exonerativos (cuando los electores no hacen responsable al gobierno de determinadas condiciones adversas), cálculos intertemporales (cuando perciben que decisiones impopulares pueden dar resultados positivos más adelante), etc. (op cit: 43).

Esta idea del voto como instrumento de control está expuesta a objeciones y críticas diversas. La más habitual suele insistir en que el voto es un instrumento romo que no permite distingos entre los diversos apartados de la acción de gobierno (op cit: 49). El problema es que votar por separado, pongamos por caso, las políticas sociales y la política fiscal daría lugar a previsibles inconsistencias. De suerte que, salvo que el sistema electoral permita establecer algún orden de preferencia (mediante segundos votos, p.e.), la discusión suele derivar hacia fórmulas capaces de afinar la función de control y, más concretamente, hacia las llamadas agencias de control (op cit: 50). Estas agencias tienen como tarea principal mejorar la capacidad de la opinión pública a la hora de juzgar la situación de la economía, el papel de los medios, etc. 
Cualquier intento de entender la mecánica del voto al margen de las condiciones en que opera la opinión pública resulta sumamente arriesgado, tal como se puede comprobar en alguno de los estudios que aparecen en este mismo volumen (Przeworsky et al 1999). En uno de ellos, podemos encontrar un intento de explicar la dis/continuidad de los gobiernos por la evolución de variables económicas (variaciones del PNB, empleo, etc.) en más de cien países durante varias décadas, sin llegar a encontrar resultados estadísticamente significativos (Cheibub y Przeworsky, 1999). ¿Se puede deducir, como sugieren los autores, que los gobiernos no se les hace responsables de su gestión de la economía o que no pagan por ello? Más razonable parece suponer que estas comparaciones «urbi et orbe» pasan por alto condiciones mínimas para el cumplimiento de este tipo de hipótesis, tales como la existencia de una opinión pública debidamente informada y capaz de discernir sobre los datos económicos.

El problema queda más claro en el estudio que Susan Stokes (1999) hace de la experiencia de varios países sudamericanos (Argentina, Perú...) donde partidos que se presentaron a las elecciones con un programa hicieron lo contrario de lo prometido y consiguieron la reelección por ese camino. Es evidente que los electores estaban mal informados sobre las opciones que se les presentaban, toda vez que algunos líderes optaron por prometer lo que los electores querían escuchar (mientras se preparaban para hacer lo contrario), en lugar de ser rigurosos y arriesgarse a perder las elecciones (como le ocurrió a Vargas Llosa frente a Fujimori).

Pero, por lo general, la capacidad de manipulación de los partidos no llega tan lejos. José M. Maravall (1999) aprovecha la experiencia de la etapa socialista española para ilustrar el repertorio de procedimientos al alcance de los gobiernos a la hora de eludir costes electorales. Estos costes suelen proceder de la persistente tendencia de los partidos a desviarse de los deseos del votante medio. ¿Por qué habrían de hacerlo, si saben que con ello arriesgan su reelección? Maravall ofrece varias respuestas: detrás de cada gobierno suele haber un partido cuyos intereses difieren sistemáticamente de los del votante medio, desde el momento en que «los militantes no se reclutan entre los votantes de centro» (op cit: 165). Esta primera dificultad suele conjurarse mediante organizaciones fuertemente centralizadas y jerarquizadas, pero esto solo hace que trasladar el problema de nivel (hacia la dirección de los partidos), especialmente si, como ocurre en el caso español, los dirigentes tienen capacidad para elaborar listas electorales cerradas y bloqueadas (op cit: 167).

Por el lado de la oposición, tampoco la representación del votante medio está asegurada, especialmente si tenemos en cuenta el extraordinario protagonismo que los medios han adquirido en la tarea de control del gobierno, lo que lleva a Maravall a la conclusión de que la oposición sigue, más que lidera, la pauta que marcan los medios (op cit: 162). A lo que habría que añadir que si alguna función de representación cabe atribuir a la prensa sería la del lector medio, en ningún caso la del votante medio.

El referéndum de la OTAN y el manejo de los primeros escándalos de la etapa socialista proporcionan a Maravall buenos ejemplos de los procedimientos empleados para lidiar con una opinión pública todavía poco experimentada. Pero 
Maravall exagera las posibilidades de manipulación cuando sugiere que Felipe González utilizó las políticas sociales para «compensar» el coste de una gestión económica poco brillante (en el mejor de los casos, el argumento puede valer para la primera mitad de la etapa socialista, pero no para la segunda). El argumento tiene dos partes: en la primera, Maravall trata de disociar la marcha de la economía (incluida la percepción de la situación económica) y los resultados electorales, poniendo el énfasis en la pésima situación de 1993, cuando Felipe González ganó por última vez, y «la fuerte recuperación económica» de 1996, cuando perdió (op cit: 186). En la segunda, presenta las políticas sociales como si de una premeditada concesión se tratara (op cit: 190).

Respecto a lo primero, el partido socialista perdió las elecciones de 1996, entre otras cosas, porque en ningún momento llegó a persuadir a la opinión pública de «la fuerte recuperación económica» a que se refiere Maravall (y no tanto por los escándalos, como apunta: 177 y 191; véase, por comparación, Caínzos y Jiménez 2000). Respecto a lo segundo, Maravall pasa por alto las tensiones en el seno del gobierno entre liberales y guerristas y la movilización de los sindicatos en favor de las políticas sociales (González 1997; Hamann 2000).

En último término, si los gobiernos de Felipe González no estuvieron sujetos a un mayor control no fue solo por la capacidad de su presidente y sus asesores más próximos para eludirlo, sino también por la ineficacia de la oposición (especialmente en la primera mitad de la etapa socialista). Pero lo importante aquí es que, tal como hace Maravall, resulta poco menos que imposible entender la función de control democrático sin poner en relación las estrategias de los partidos con la dinámica de la opinión pública.

\section{EL MITO DE LA COMPLACENCIA}

En ocasiones, los analistas han creido encontrar una correspondencia directa entre el curso de la opinión pública y la capacidad de respuesta de los políticos, tal como se desprende del análisis del caso norteamericano que aparece en esta misma compilación (Stimson 1999). A juzgar por este autor, el caso norteamericano sería un paradigma de feliz correspondencia entre la opinión pública y una clase política dispuesta al cumplimiento. La reciente obra de Jacobs y Shapiro (2000) ofrece, en cambio, un cuadro mucho menos idílico que el sugerido por Stimson en lo que se refiere a la capacidad de cumplimiento de la clase política norteamericana. Merece la pena detenerse en el estudio de Jacobs y Shapiro, pero antes me gustaría hacer un breve recordatorio de por qué el caso norteamericano resulta de especial interés.

No hace falta recordar la abrumadora presencia de las encuestas en EEUU, baste con decir que se calcula por decenas de millones el número de entrevistas anuales, de suerte que el americano medio tiene la oportunidad de ser entrevistado repetidas veces a lo largo de su vida (Fishkin 1995). Pero lo significativo no es tanto la magnitud de este fenómeno como el sesgo plebiscitario característico de la democracia americana. Las primeras referencias a esta peculiaridad datan de finales del siglo XIX, cuando la democracia americana se perfilaba como una 
variante de, o un estadio ulterior en el desarrollo democrático, caracterizable como el «gobierno de la opinión pública» (op cit: 71 ss). Lo que por entonces podía parecer solamente una peculiaridad idiosincrásica o una tendencia tomó cuerpo medio siglo más tarde con la aparición de Gallup y la irrupción de los sondeos electorales.

El escrutinio de la opinión pública alcanzó tanta notoriedad que Hollywood comenzó a explotar el tema apenas diez años después del primer sondeo de Gallup (1936), con la película Magic Town (1947). En la película, un aventajado demóscopo interpretado por James Stewart se valía de una pequeña ciudad del medio oeste (Grandview), capaz de reproducir en microcosmos el clima de opinión del país entero, para hacer predicciones poco costosas sobre cualquier tema de interés nacional. Por entonces la demoscopia ya se había convertido en otra forma más de negocio, pero no conviene olvidar que la idea primera de Gallup era poner las encuestas al servicio de la democracia y, más concretamente, dar respuesta a los interrogantes acerca del voto. «¿Cómo podemos saber si el público vota por el candidato o por su programa? ¿Cómo podemos saber si apoya todos los puntos del candidato o si está a favor de algunos y en contra de otros?» (Gallup 1939, citado por Fishkin 1995: 77).

Nada más lejos, a primera vista, del optimismo reformista de Gallup que la presente discusión sobre la política americana de los noventa, discusión que presenta un cierto carácter paradójico: pues nunca se habían hecho tantos sondeos como se han hecho en la década de los noventa y, sin embargo, nunca la ciudadanía se ha sentido tan distante y enajenada respecto de la clase política. La práctica totalidad de los indicadores de confianza democrática han registrado un deterioro imparable desde los años sesenta que, salvo contados momentos de recuperación (coincidiendo con las reelecciones de Reagan y Clinton), han llevado a la política americana a una especie de anemia democrática (Norris 1999). Por supuesto, no se trata de culpar a los sondeos, pero no deja de ser curioso que se haya llegado a esta situación en el momento justo en que los asesores de Clinton realizaban sondeos con carácter semanal e incluso diario.

El estudio de Jacobs y Shapiro (2000) lleva un subtítulo sumamente explícito: «Political Manipulation and the Loss of Democratic Responsiveness» ${ }^{1}$. El libro empieza con un ataque a lo que los autores denominan el «mito de la complacencia», en virtud del cual es habitual entre comentaristas políticos e incluso académicos la presunción de que los líderes han perdido protagonismo en favor de asesores y consultores que les informan puntualmente de los deseos populares. Los líderes habrían traicionado así lo que tienen de específico y se limitarían

1 El primer problema que se deriva de este subtítulo es la traducción de responsiveness. Aunque algunos autores han sugerido traducirlo por cumplimiento, esta opción plantea el problema de que encaja bien con la idea de la democracia como mandato, pero no tanto con la idea de confianza y control. Pues así como el mandato plantea un problema de cumplimiento, la idea de control plantea un problema de atención y acomodación de los políticos respecto a las preferencias de los electores. Desde este punto de vista, podría traducirse por complacencia, lo cual resulta más en consonancia con el título -irónico- del libro: «Los políticos no se doblegan» («Politicians don’t Pander»). Por supuesto, hay otras opciones no menos satisfactorias, tales como receptividad o sensibilidad. Utilizaré una u otra siguiendo un criterio de oportunista conveniencia. 
a seguir la corriente de la opinión pública, en lo que sería una perniciosa suplantación de la política con mayúsculas por el simple oportunismo. El mito estaría representado de manera paradigmática por la relación entre Clinton y Dick Morris durante los meses previos a la reelección del primero.

Por su parte, Jacobs y Shapiro argumentan que: a) la receptividad de la clase política norteamericana respecto a las preferencias de los electores ha declinado durante los años ochenta y noventa; y b) que esto ha sido así pese a severas derrotas electorales tanto republicanas como demócratas, lo que les hace suponer que «el castigo electoral puede no ser suficiente para mejorar la influencia del público sobre el gobierno» (op cit: xvii). Conviene no perder de vista ambas partes del argumento.

Podemos identificar varios factores causantes del declive de la receptividad democrática:

a) la polarización ideológica de los partidos, lo que aumenta el coste de decisiones políticas a favor del votante medio (op cit: 29 ss).

b) Fraccionamiento político y parlamentario relacionado, entre otras cosas, con los cambios en la financiación de las campañas y el creciente protagonismo de las fuentes exógenas de financiación (lobbies, grupos de interés, etc.), lo que debilita la capacidad disciplinaria de los partidos.

c) Fraccionamiento electoral derivado del doble tipo de elecciones característico de la política norteamericana, de suerte que los políticos tienen que atender dos segmentos distintos del electorado: «la estrecha franja de partidarios leales necesarios para ganar las primarias y el más amplio grupo de votantes volátiles que se mueven entre los partidos y que son necesarios para ganar elecciones generales» (op cit: $11 \mathrm{ss}$ ). No hay que decir que aunque son estos últimos, los más moderados, los que tienen la última palabra en elecciones generales, los primeros, los más ideologizados, son decisivos en la financiación de las campañas.

Hay que tener en cuenta, por otro lado, que más que una tendencia lineal, se trata de una tendencia condicionada por la distancia respecto de las elecciones, de manera que dentro de cada legislatura se puede observar una recuperación de la complacencia y la receptividad de los partidos a medida que se acerca el momento electoral.

Los políticos tienen dos maneras de responder ante la opinión pública: una consiste en recabar información de la opinión pública para diseñar las políticas de acuerdo con las preferencias del votante medio. Es lo que se conoce coloquialmente como pandering, una expresión estigmatizada por la presunción de falta de principios y oportunismo, pero que, a juicio de los autores, no hace sino reflejar «el miedo, el malestar y la hostilidad de las élites hacia el consentimiento y la influencia del público sobre los asuntos de gobierno» (op cit: xiv).

La segunda manera consiste en estudiar la opinión pública para escoger «las palabras, los símbolos y los argumentos más seductores para ganarse a la opinión pública a favor de sus políticas preferidas (...) Su objetivo es simular complacencia (...) En su intento por reducir los costes de subordinar las preferencia de los electores a las suyas propias, los políticos usan sondeos y focus groups no para acercar sus posiciones a las del público sino para todo lo contrario» (op cit: xv). 
Los políticos disponen de tres mecanismos para modificar la opinión pública en su favor: los medios, los sondeos y las estrategias de comunicación. En este artículo, me centraré en el papel de los sondeos. De acuerdo con lo expuesto, caben dos posibilidades: que los sondeos sean un factor, si no decisivo, sí al menos contributivo en el diseño de las políticas o que, por el contrario, se utilicen exclusivamente para que las políticas, diseñadas al margen de la opinión pública, alcancen la máxima aceptación posible entre el público. Doy por supuesto que esta segunda opción implica alguna forma de manipulación, toda vez que los políticos tratan de llevar al público a conclusiones distintas de las que llegaría si dispusiera de la información adecuada.

\section{EL PAPEL DE LOS SONDEOS}

Para ilustrar el papel de los sondeos en el proceso de toma de decisiones, podemos recurrir a la comparación de dos experiencias distintas en el primer mandato de Clinton (1993-96): el macroproyecto de reforma sanitaria de 199394 y las microrreformas fiscales y sociales de 1995-96. La primera era una promesa electoral que se abordó nada más iniciarse el mandato. Las segundas se plantearon en la segunda mitad del mandato, cuando se acercaban nuevas elecciones.

Como se recordará, Bill Clinton llegó a la Casa Blanca en situación precaria (43\% del voto, el segundo porcentaje más bajo del siglo), en buena medida consecuencia del éxito sin precedentes de Ross Perot, que consiguió el mejor porcentaje jamás registrado por un tercer partido (19\%). George Bush consiguió el $37 \%$ restante, tras una de las campañas más desgraciadas de la historia electoral norteamericana (llegó a tener un $90 \%$ de popularidad tan solo un año antes de las elecciones).

No es de extrañar, por tanto, la facilidad con que Clinton sustituyó sus promesas iniciales de rebajas fiscales para la clase media por un plan riguroso de reducción del déficit público, que había sido el caballo de batalla de Perot. Pero esto no hizo sino más imperiosa la necesidad de una propuesta alternativa que sirviese a un tiempo para visualizar el carácter distintivo de un presidente demócrata (tras doce años de reaganismo) y para estimular el crecimiento económico.

Aunque Jacobs y Shapiro enfatizan esta segunda dimensión, afirmando que «Fue primeramente el compromiso de Clinton con el crecimiento económico y la reducción del déficit lo que le llevó a apoyar un seguro universal de salud» (op cit: 80), la iniciativa encajaba bien en el ideario del staff de la Casa Blanca, empezando por Hillary Clinton, poco sospechosa de economicismo ${ }^{2}$, que se dispuso enseguida a encabezar la reforma.

Las peripecias de la iniciativa son bien conocidas y no es cosa de repetirlas aquí (véase, por ejemplo, Skocpol 1996). Baste con recordar las principales

2 De acuerdo con David Gergen, asesor de Clinton durante la primera fase del mandato, Hillary Clinton solía alinearse con la rama populista del staff: «No hemos venido aquí a gastar todo nuestro tiempo cortando los déficits creados por los republicanos» era una de sus frases preferidas $(2000,277)$. 
razones del fracaso de esta iniciativa que estaba llamada a desempeñar un papel estelar en la ejecutoria de Clinton, pero que naufragó en medio de un cúmulo de errores:

a) Errores de apreciación, en primer lugar, sobre la capacidad del presidente para vender iniciativas. A la altura de los noventa, los presidentes llevaban largo tiempo sometidos a una erosión continuada de su autoridad real y simbólica, pese a lo cual el staff de la Casa Blanca persistió en la idea de que Clinton podía vender cualquier iniciativa por compleja que fuera (Gergen, 2000: 304).

b) Errores de apreciación sobre la capacidad de los grupos de interés (en este caso, la asociación de aseguradoras médicas), convertidos a la sazón en criptopartidos no susceptibles de control por el público (la expresión procede de Johnson y Broder 1996, citada por Gergen 2000: 305)

c) Errores de manejo parlamentario en un Congreso fraccionado donde los partidos carecían de capacidad de disciplina sobre sus miembros.

d) Errores de interpretación de los valores expresados por la opinión pública, tras décadas de deterioro de la confianza en la capacidad del gobierno.

¿De qué sirvieron los sondeos en este caso? La propia dinámica de la iniciativa dejaba poco margen, en principio, para los sondeos. Por un lado, se trataba de una promesa electoral que había madurado en el contexto de la estrategia de campaña. Por otro, se trataba de un tema demasiado complejo para dejarlo al albur de la opinión pública. La iniciativa fue promovida a través de una task force que buscó un doble objetivo: conseguir consenso en un entorno sumamente fraccionado en el que se debatía sobre un largo repertorio de modelos de gestión sanitaria y conseguir credibilidad ante la opinión pública (Jacobs y Shapiro 2000: 86).

Es claro que los rasgos generales de la iniciativa gozaban del apoyo de la opinión pública y que esta reconocía la prioridad del asunto, pero en ningún caso puede decirse que la opinión pública influyese en el diseño de la política. Por el contrario, el responsable de los sondeos (Stanley Greenberg) ha reconocido explícitamente que las preferencias de la mayoría fueron sacrificadas a los objetivos partidarios y que él mismo quedó marginado del proceso decisorio (op cit: 96-97).

Temeroso de que la opinión pública fuese incapaz de discernir sobre los detalles, el staff de la Casa Blanca depositó toda su confianza en la capacidad del presidente para vender la iniciativa mediante una estrategia de priming. En consecuencia, los sondeos y focus groups estuvieron exclusivamente orientados a diseñar no la iniciativa sino la presentación pública de la misma en tres de sus aspectos: la elección del eslogan general (seguridad para todos), el énfasis en los beneficios individuales de la reforma y la selección de los términos más adecuados para describirla. La recomendación de Greenberg consistió, en este caso, en subrayar las dimensiones de seguridad y beneficio personal, sin entrar en detalles y evitando referencias a grupos sociales que, aún siendo destinatarios de la reforma (minorías, inmigrantes...), podían entrar en colisión con los intereses del votante medio (op cit: 109 ss).

Como es bien sabido, de poco sirvió la implicación directa y personal del presidente y la primera dama en defensa de la iniciativa. Los grupos de interés 
reaccionaron con una auténtica inundación de propaganda (los famosos «Harry and Louise» ${ }^{3}$ ) que respondía a la iniciativa con una sencilla sugerencia: big government. Por supuesto, también en este caso los sondeos jugaron un papel en la identificación de una ambivalencia profunda respecto a la reforma: acuerdo con los objetivos generales mezclado con desconfianza respecto a la eventual burocracia encargada de implementarlos. Aunque los Clinton trataron de contrarrestar el efecto de la campaña con una versión propia de «Harry and Louise», el priming de los oponentes a la reforma resultó más eficaz. Lo irónico del asunto es que, consciente de los peligros que amenazaban la iniciativa, el staff de la Casa Blanca había optado por un modelo de competencia que minimizaba el papel de la administración.

En cualquier caso, el episodio de la reforma sanitaria constituye un buen ejemplo de proceso decisorio al margen de los sondeos, cuya función parece adaptarse bien a la prevista por Jacobs y Shapiro: medir la distancia entre los objetivos prefijados y el votante medio e identificar los procedimientos para que el votante medio se acerque a los objetivos, evitando en lo posible el coste de la desviación. El problema es que, tal como acabamos de ver, el coste de la desviación puede ser no solamente electoral (que lo fue, dando lugar a una mayoría republicana sin precedentes en las elecciones legislativas de 1994), sino que puede comprometer la propia viabilidad de la iniciativa, tal como ocurrió con la reforma sanitaria.

En el ambiente de frustración derivado del fracaso de la reforma sanitaria, Clinton perdió el control del congreso en las elecciones legislativas de 1994, dando paso a la «revolución conservadora» liderada por Newton Gingrich (nuevo presidente de la cámara baja). Aunque el citado Stanley Greenberg se ha referido a la victoria republicana de 1994 como una working-class revolt contra los demócratas (Greenberg 1997: 288), conviene aclarar que no tuvo nada que ver con el fenómeno de los Reagan Democrats de los ochenta, cuando los trabajadores manuales pasaron a votar mayoritariamente republicano, sino que se trató más bien de un movimiento abstencionista. De hecho, Clinton recuperó el apoyo de estos votantes en las elecciones de 1996 (op cit: 285).

Pero la barrida electoral de 1994 dejó a Clinton contra las cuerdas en el ecuador de su primer mandato. En ese momento entra en escena Dick Morris, antiguo asesor de Clinton en su época de gobernardor de Arkansas. Morris comienza su tarea proponiendo una estrategia de «triangulación» consistente en someter los puntos clave de la agenda conservadora (tal como habían sido proclamados en el «Contrato con América») al criterio de la opinión pública, filtrando los que contasen con la aprobación del votante medio y proponiendo soluciones en los términos más aceptables para el electorado demócrata (Morris, 1999: 37-384).

Nada más lejos, por tanto, del estilo de gobierno que pudimos observar en la primera parte del mandato. Frente al dramatismo de las grandes iniciativas de cor-

3 En el anuncio, una pareja de americanos medios se lamentaba de que el gobierno les forzase a escoger entre unos pocos planes diseñados por burócratas, hasta llegar a la conclusión de «tiene que haber otra manera mejor».

4 Según el propio MoRris, la mencionada estrategia estuvo inspirada en la manera como Mitterrand maniobró durante su cohabitación con el primer ministro Chirac (op cit: 37). 
te tecnocrático y la consiguiente reacción de los medios y los grupos de interés, Morris opuso una política incrementalista, paso a paso, centrada en iniciativas más o menos puntuales que Clinton debía integrar dentro de un argumento típico de la tercera vía: una combinación de responsabilidad republicana con igualdad de oportunidades al estilo demócrata (op cit: 13, 86, 92, 208 y appendix 581).

Por supuesto, tampoco esta estrategia estuvo exenta de costes. Si la estrategia anterior resultaba sumamente vulnerable desde el punto de vista electoral, esta segunda hubo de afrontar costes políticos inmediatos, comenzando por la propia reacción del staff de la Casa Blanca, que veía en ella una traición a las esencias del Partido Demócrata, al tiempo que las filas republicanas denunciaban el oportunismo de Clinton (Jacobs y Shapiro, 2000: 278 y 281).

Podemos resumir las líneas básicas de la nueva estrategia, tal como resultó de los sondeos, de la siguiente manera: a) aprobación de las propuestas republicanas en lo relativo a recorte de impuestos, reforma de las políticas anti-pobreza, reducción del déficit y reducción de la burocracia federal 5. b) Rechazo de los recortes a los programas sociales, sanitarios y educativos, así como a la protección medioambiental (Morris 1999: 93).

Aunque la «triangulación» ha sido frecuentemente minimizada como una simple vía intermedia, los sondeos dieron lugar a propuestas cualitativamente distintas de las inicialmente contempladas. Un buen ejemplo lo constituye la política fiscal: ante el dilema típico de recorte generalizado de impuestos (al estilo republicano) o recorte para las clases medias (al estilo demócrata), las propuestas más apoyadas por los sondeos resultaron ser los recortes asociados a acciones concretas (p.e., mandar a los hijos a la universidad). Esto dio lugar a una política de créditos fiscales a la que Morris se refiere como triangulación funcional, por oposición a la retórica clasista con que tradicionalmente se presenta la política fiscal de corte demócrata (op cit: 82, 85 y 223).

Pero donde los sondeos resultaron más decisivos fue en la reforma de las políticas anti-pobreza. En este punto, Clinton abandonó sus posiciones previas y aceptó el requerimiento de trabajo como condición para el cobro de subsidios (apoyado por el $73 \%$ de los encuestados, frente al 23\%) y limitaciones temporales para el mismo (55\% frente a 39\%), así como recortes masivos a los inmigrantes ilegales (aun cuando los sondeos habían recogido una situación de empate técnico: 50\% frente a 47\%) (op cit: appendix 384-385, 466-467).

En el verano de 1996 Clinton aprobó la controvertida reforma del welfare con el voto en contra de la mitad de los congresistas demócratas y la radical oposición del staff y del gabinete de la Casa Blanca. En un panel reciente sobre el legado de Clinton, el citado Dick Morris compara la citada reforma de Clinton con la apertura de relaciones con China protagonizada por un anticomunista conspicuo como Nixon ${ }^{6}$. Es pronto para saber si la historia recordará a Clinton por su reforma del welfare y, tal como sugiere Morris, por haber conseguido que el tema de la infraclase dejase de ser un tema político y electoral. Pero lo que sí

5 En ese momento, Clinton había hecho ya progresos significativos en los dos últimos apartados, con reducciones del 60\% y del 10\%, respectivamente (MorRIs 1999: 93).

6 «What we'll remember», Time. Nov. 202000. 
sabemos es que ese fue el tipo de decisión que desarmó a la oposición republicana y facilitó a Clinton la reelección pocos meses después (no hay que olvidar que la medida estuvo acompañada de otros acuerdos bipartidistas sobre aumento del salario mínimo, mejora de la cobertura sanitaria, etc.).

El contraste entre la experiencia de la macrorreforma sanitaria de 1993-94 y las microrreformas de 1995-96 no puede ser más elocuente: en el primer caso, una formulación del problema en términos estrictamente técnicos y partidarios dio lugar al naufragio legislativo, primero, y a la debacle electoral, poco depués. En el segundo, un esfuerzo de aproximación y sensibilidad hacia el votante medio, aprovechando el impulso de la contraofensiva republicana, dio lugar al desarme de la oposición y a la consiguiente reelección. En el primer caso, el papel de los sondeos estuvo ciudadosamente limitado a la presentación de la iniciativa, a fin de conducir al público a la aprobación de la misma. En el segundo, los sondeos estuvieron en el centro mismo de la toma de decisiones.

\section{LA PARADOJA DEMOCRÁTICA: ¿QUIÉN SIGUE A QUIÉN?}

Recordemos la distinción de Jacobs y Shapiro sobre las dos maneras como los políticos pueden responder a la opinión pública: una consiste en recabar información de la opinión pública para diseñar las políticas de acuerdo con las preferencias del votante medio. La segunda manera consiste en estudiar la opinión pública no para ajustarse a ella, sino para simular complacencia con ella. Parecería que, como resultado de la comparación entre las diversas experiencias del primer mandato de Clinton, hubiéramos encontrado buenos ejemplos de las dos maneras a que se refieren Jacobs y Shapiro, pero en realidad no hemos hecho más que tropezarnos con la paradoja con que empezábamos este artículo: los líderes están para dirigir, pero se espera de ellos que no se aparten de los deseos populares. De manera que si son realmente líderes, deberán tener cuidado en no parecer que obran de espaldas al público. Si, por el contrario, optan por complacer las preferencias del público, deberán tener cuidado en no parecer seguidores oportunistas.

Dick Morris se esmera en dejar claro que Clinton no pertenece a estos últimos. En manos de Clinton, los sondeos son «una herramienta de gobierno, una técnica para facilitar el progreso de la democracia». En algún momento, Morris recurre a la metáfora del navegante que utiliza los sondeos para captar el sentido del viento y determinar la corriente más adecuada para llegar a su destino. El navegante vira sucesivamente a derecha y a izquierda, pero es solo con el fin de alcanzar el «punto medio». Clinton, según Morris, «siempre llega allí» (1999: 84).

Pero la metáfora preferida de Morris es la del diálogo: como nuevo demócrata, Clinton no quiso sujetarse a ningún dogma partidario y utilizó los sondeos para testar sus propuestas y abrir un diálogo persuasivo con el público. Pero «nunca usó los sondeos para determinar qué posición debía tomar en un tema. Nunca (Clinton) usó los sondeos principalmente para escoger cuáles de sus posiciones eran populares. Cuando los sondeos indicaban que su posición en un tema 
era impopular, él solía pedir un estudio sobre cómo convencer a la gente de su punto de vista» (op cit: 338 ).

En su celo por contrarrestar la acusación de la prensa de que Clinton estaba gobernando a golpe de encuesta, Morris llegó a elaborar una lista de asuntos en los que Clinton había tomado decisiones impopulares (varios de ellos en materia de política exterior). La ironía de esa lista es que formaba parte de un informe demoscópico sobre la valoración de los logros del primer mandato de Clinton, así como la agenda de temas de un eventual segundo mandato ipor orden de popularidad! (op cit: appendix 581-83).

\section{6. ¿ PODEMOS SACAR CONCLUSIONES?}

Es difícil sacar conclusiones de una sola experiencia, pero tampoco se trata de una experiencia cualquiera. Para bien o para mal, el paso de Bill Clinton por la Casa Blanca ha sido objeto de atención cuidadosa por múltiples razones. Más aún, su actuacion ha sido un referente obligado para todos aquellos que siguen con interés las vicisitudes de la llamada tercera vía. Si tal proyecto llega a alguna parte, lo único seguro es que su destino no va a ser ajeno a lo que hagan los nuevos demócratas americanos que le dieron origen.

Uno de los propósitos de este artículo era contrastar la tesis central que articula el trabajo de Jacobs y Shapiro: frente a la visión ingenua en virtud de la cual la democracia americana parecía haber llegado a un punto de feliz encuentro entre la clase política y la opinión pública, estos autores aportan impresionante evidencia de que el comportamiento de los partidos en la primera mitad de los noventa no se correspondía en modo alguno con esa visión. De hecho, la evolución política de EEUU desde los años sesenta y en particular durante los años ochenta parecía empujar en dirección contraria: hacia la polarización y el distanciamiento con respecto al votante medio. Clinton trató de responder a ello desde su primer llamamiento de campaña ( «Putting People First»), pero tampoco él pudo escapar fácilmente de las tendencias que habían dominado a los partidos durante treinta años.

Lo significativo del caso es que, tras un periodo de aprendizaje tanto propio (la derrota de su iniciativa de reforma sanitaria y la consiguiente debacle electoral de 1994) como ajeno (la victoria republicana del «Contrato con América» y la subsiguiente incapacidad para administrarlo), Clinton encontró finalmente el camino de la complacencia. Cabe preguntarse si estamos ante un caso anómalo o, por el contrario, ante una nueva pauta de receptividad democrática. A nadie se le escapa que los proyectos asociados a la tercera vía muestran una particular disposición a la complacencia con el votante medio -no en vano suelen presentarse con la pretensión de centrismo. Pero no conviene olvidar dos de las condiciones que hicieron posible la extraordinaria plasticidad de Clinton: una larga tradición de gobierno de la opinión pública y un sistema político caracterizado por la debilidad de los partidos y su absoluta incapacidad para imponer disciplina sobre sus miembros (justamente las dos cosas a las que Jacobs y Shapiro no han prestado la suficiente atención). Esto puede dificultar la eventual exportación de este tipo de experiencias. 
Una cosa parece, sin embargo, al margen de toda discusión: el papel estelar de los sondeos en el día a día de la democracia. La experiencia de Clinton nos ha proporcionado una excelente ilustración de las dos maneras posibles de utilizarlos. Si se me permite la licencia, me gustaría terminar un poco al modo antiguo: «por sus sondeos les conoceréis».

\section{REFERENCIAS BIBLIOGRÁFICAS}

CAínzos y JiMÉNEZ (2000): "El impacto de los escándalos de corrupción sobre el voto en las elecciones generales de 1996". Historia y política, 4, pp. 93-133.

Cheibub y PrZewORSKy (1999): «Democracy, Elections and Accountability for Economic Outcomes», en Przeworsky, Stokes y Manin: «Democracy, Accountability, and Representation», Cambridge University Press, pp 222-249.

FishKIN, James (1995): «The Voice of the People. Public Opinion \& Democracy», Yale University Press.

GalluP, George (1939): «Public Opinion in a Democracy». The Stafford Little Lectures, Princenton University Extension Fund.

GERGEN, David (2000): «Eyewitness to Power», Simon\&Shuster.

GonZÁLEZ, J.J. (1997): «Classes, Citizens, and Classes of Citizens. The Electoral Cycle of Post-Socialism (1986-94)», Revista Española de Investigaciones Sociológicas, English Edition 1997, pp 125-155.

GreEnberG, Stanley (1997): «Popularizing Progressive Politics», en Greenberg y Skocpol: «The New Majority. Toward a PopularProgressive Politics», Yale University Press, pp 264-298.

HamanN, Kerstin (2000): «Linking Policies and Economic Voting. Explaining Reelection in the Case of the Spanish Socialist Party», Comparative Political Studies, Vol. 33 No 8, 1018-1048.

JACOBS y SHAPIRO (2000): «Politicians Don't Pander. Political Manipulation and the Loss of Democratic Responsiveness», The University of Chicago Press.

JoHnSON y BRODER (1996): «The System: The American Way of Politics at the Breaking Point», Boston, Little Brown.

MANin, PRZEWORSKY y Stokes (1999): «Elections and Representation», en Przeworsky, Stokes y Manin: «Democracy, Accountability, and Representation», Cambridge University Press, pp 29-54.

MaraValL, J.M. (1999): «Accountability and Manipulartion», en Przeworsky, Stokes y Manin: «Democracy, Accountability, and Representation», Cambridge University Press, pp 154-196.

MorRIS, Dick (1999): «Behind the Oval Office. Getting Reelected against All Odds», Renaissance Books.

NoRrIs, Pippa (1999): «The Growth of Critical Citizens?», en Norris, Pippa: «Critical Citizens. Global Support for Democratic Governance», Oxford University Press, pp $1-27$.

SкостоL, Theda (1996): «Boomerang: Clinton's Health Security Effort and the Turn agaisnt Government in US Politics», NY, W W Norton.

SKOCPOL, Theda (1997): «A Partnership with American Families», en Greenberg y Skocpol: «The New Majority. Toward a PopularProgressive Politics», Yale University Press, pp 104-129. 
Stimson, James (1999): «Party Government and Responsiveness», en Przeworsky, Stokes y Manin: «Democracy, Accountability, and Representation», Cambridge University Press, pp 197-221.

StokeS, Susan (1999): «What Do Policy Switches Tell Us about Democracy?», en Przeworsky, Stokes y Manin: «Democracy, Accountability, and Representation», Cambridge University Press, pp 98-130.

\title{
RESUMEN
}

La democracia está plagada de paradojas: si los líderes se apartan de los deseos populares, son acusados de despotismo; si, por el contrario, se pliegan a ellos lo serán de complacencia y demagogia. Los analistas discuten sobre la capacidad del voto como instrumento de control, al tiempo que los políticos se afanan en eludirlo. Los políticos se sirven para ello de los sondeos, pero los sondeos reciben muy poca atención desde este punto de vista.

El primer mandato de Clinton en la Casa Blanca (1992-1996) ilustra bien esta serie de paradojas. La experiencia pionera de la tercera vía nos permite no solo reflexionar sobre ellas, sino también ilustrar los posibles escenarios. Frente a la polarización ideológica que caracterizó la política americana desde la segunda mitad de los sesentas hasta su primer mandato, Clinton pareció encontrar finalmente el camino de la complacencia que le llevó a la reelección de 1986. Cabe discutir si Clinton abrió con ello una nueva era de receptividad democrática, pero hay pocas dudas sobre su extraordinaria influencia en el uso de los sondeos.

\begin{abstract}
Democracy is riddled with paradoxes. If the leaders stray away from the people's wishes, they are accused of despotism; if, on the other hand, they bow to the people's will, they are accused of pandering. Analysts argue about the vote's role as an instrument of control while politicians strive to circumvent this control. Politicians use polls for this purpose, but little attention is paid to this aspect of polls.

Clinton's first term in the White House (1992-1996) is a good illustration of this series of paradoxes. The pioneer experience of the third way allows not only to reflect upon these paradoxes but to explain possible scenarios. Faced with the ideological polarization that characterized American politics from the second half of the sixties to his first term, Clinton finally seemed to have found the path of pandering that got him reelected in 1996. Whether or not Clinton inaugurated a new era of democratic responsiveness is open to discussion, but there can be little doubt about its extraordinary influence on the use of polls.
\end{abstract}

